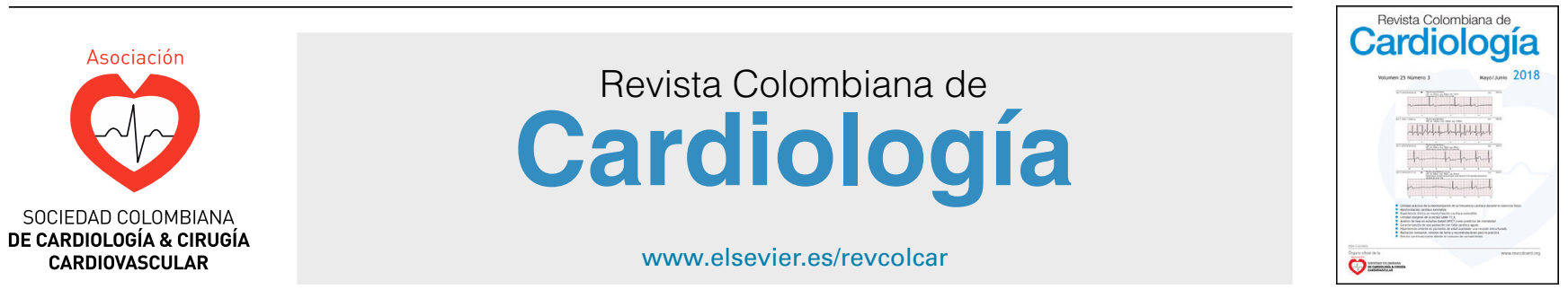

ARTÍCULO ESPECIAL

\title{
Salud mental de los cardiólogos intervencionistas: Estrés ocupacional y consecuencias mentales de la exposición a radiación ionizante
}

\author{
Diana Restrepo
}

Universidad CES, Medellín, Colombia

Recibido el 13 de mayo de 2019; aceptado el 1 de diciembre de 2019

\section{PALABRAS CLAVE \\ Radiación ionizante; Trastornos mentales; Agotamiento profesional; Suicidio}

\section{KEY WORDS}

Ionising radiation; Mental disorders; Professional burn-out; Suicide

\begin{abstract}
Resumen Este documento centra su atención en los riesgos para la salud mental a los que se enfrentan los cardiólogos intervencionistas como consecuencia de la exposición al estrés laboral que les impone el ejercicio de la profesión, como también, al riesgo derivado de la exposición crónica a bajas dosis de radiación ionizante. El agotamiento profesional, o "burnout", es la principal consecuencia psicosocial que enfrenta el cardiólogo intervencionista, seguida de la depresión mayor, la dependencia a sustancias y el riesgo suicida, este último mayor en médicos que en la población general. En cuanto a la radiación ionizante, la investigación es aún incipiente y ha mostrado una reducción significativa en la fluencia y la memoria verbal, habilidades del hemisferio izquierdo que se verían comprometidas con la exposición a bajas dosis de radiación. Resulta prometedor el estudio de la desregulación de microARN en cardiólogos intervencionistas, puesto que por la especificidad tisular que tienen, son biomarcadores ideales para explorar los mecanismos moleculares que subyacen y están asociados con exposición a bajas dosis de radiación. Se requiere abordar este importante tema con una mirada desde la promoción de estilos de vida más saludables y desde la prevención, que permita que los síntomas mentales se detecten temprano y el curso y pronóstico de los trastornos mentales sea mejor, además de disminuir la exposición a la radiación y promover la investigación en esta importante área de la salud mental ocupacional.

(c) 2019 Sociedad Colombiana de Cardiología y Cirugía Cardiovascular. Publicado por Elsevier España, S.L.U. Este es un artículo Open Access bajo la licencia CC BY-NC-ND (http:// creativecommons.org/licenses/by-nc-nd/4.0/).
\end{abstract}

Mental health of interventionist cardiologists: Occupational stress and consequences of exposure to ionising radiation

Abstract This article focuses on the risks for mental health that interventionist cardiologists face as result of exposure to the occupational stress they are subjected to in the practice of their profession, as well as the risk arising from the chronic exposure to low doses of ionising radiation. Professional exhaustion, or "burnout", is the main psychosocial consequence that

Correo electrónico: dianarestrepobernal@gmail.com 
the interventionist cardiologist faces, followed by major depression, substance dependence, and suicide risk. The latter is greater in doctors than in the general population. As regards the ionising radiation, studies, research, although in its infancy, has shown a significant reduction in verbal fluency and memory, as well as abilities in the left hemisphere that could be compromised with exposure to low doses of radiation. The study on the dysregulation of microRNAs in interventionist cardiologists seems promising since, due to their tissue specificity, they are ideal biomarkers in order to explore the underlying molecular mechanisms that are associated with the exposure to low doses of radiation. This important subject needs to be approached with a view to promoting more healthy lifestyles, and from the prevention, which would allow the mental symptoms to be detected earlier. The course and prognosis of the mental disorders could be improved, besides decreasing the exposure to the radiation and to promote research into this important area of occupational mental health.

(C) 2019 Sociedad Colombiana de Cardiología y Cirugía Cardiovascular. Published by Elsevier España, S.L.U. This is an open access article under the CC BY-NC-ND license (http:// creativecommons.org/licenses/by-nc-nd/4.0/).

The most tragic thing in the world is a sick doctor' ' (Bernard Shaw, "The Doctor's Dilemma") A universal truth: no health without a healthy workforce

\section{Introducción}

La Organización Mundial de la Salud, en su informe de $2013^{1}$, declaraba como una verdad universal la necesidad de contar con un equipo de salud para garantizar la cobertura universal en salud. Otros investigadores han expandido este concepto al decir que no puede haber salud sin una fuerza de trabajo saludable ${ }^{2}$.

Debido a la importancia que tiene el personal de salud para poder garantizar un desempeño excelente en un trabajo cada vez más exigente por el escenario complejo en el que se presta el servicio, la fuerza de trabajo clínica es un foco de interés creciente ${ }^{3}$.

Sin embargo, la mayor parte de esta investigación está impulsada por medidas cuantitativas que reflejan las proyecciones de demanda basadas en datos demográficos, incidencia de enfermedad y necesidad de anticipar la mano de obra clínica que se requerirá para prestar los servicios, pero solo recientemente se ha prestado atención de forma incipiente a un factor crucial: el bienestar y la salud mental del personal clínico ${ }^{4}$.

\section{Bienestar y trabajo}

Nunca antes el bienestar de la fuerza de trabajo ha sido un tema principal en el cuidado de la salud ${ }^{5}$. Esto se observa en la creciente incidencia de problemas de salud mental, como estrés, "burnout", depresión, dependencia a drogas y alcohol y suicidio ${ }^{6,7}$.

En un estudio realizado por la Asociación Médica e Investigadores de la Clínica Mayo informaron que el 54\% de los médicos en los Estados Unidos experimentaban agotamiento profesional. Las tasas de suicidio también son altas. En una revisión de cuatro décadas de estudios sobre el suicidio en los médicos se estimó que las posibilidades de morir por esta causa son más altas en los médicos que en la población general, 70\% en médicos, más en médicos hombres y 250 a $400 \%$ en las mujeres médicas ${ }^{8}$.

Este asunto es de tal importancia que para lograr el llamado "triple objetivo", esto es mejorar la salud de la población, mejorar la experiencia del paciente y reducir los costos de atención, Bodenheimer y Sinsky defendieron la importancia de agregar a esta tríada el "cuarto objetivo ", mejorar la vida laboral de los profesionales de la salud".

En el 2015 el jefe del Sistema Nacional de Salud de Inglaterra (NHS, su sigla en inglés) declaró que los niveles de estrés entre el personal del Instituto eran "asombrosamente altos" y debería tratarse como un problema de salud pública $^{10}$. A esto se suma el informe reciente de 2017 del Comité de Sostenibilidad del NHS y Adult Social Care, en el que se anuncia que la ausencia de estrategia nacional a largo plazo para asegurar adecuadamente mano de obra calificada, bien entrenada y comprometida [...] representa la mayor amenaza interna para la sostenibilidad del $\mathrm{NHS}^{11}$.

\section{Profesión médica y salud mental}

Una pregunta válida que puede formularse alguien no muy informado en este tema sería: ¿tienen los médicos mayor riesgo que la población general de sufrir problemas de salud mental? La respuesta, según la evidencia científica disponible en la actualidad, es sí; los profesionales de la medicina tienen mayor riesgo que la población general de tener problemas de salud mental. Numerosos factores individuales y ocupacionales vulneran la salud mental de los médicos; entre estos se encuentran:

1. Las altas demandas emocionales propias de la profesión médica y en particular de especialidades como la cardiología en las que los resultados de la intervención determinan claramente la posibilidad que tiene un paciente de vivir o morir ${ }^{12}$.

2. La sobrecarga resultante de intentar proveer más y mejor calidad en la atención ${ }^{13}$. 
3. La pérdida de la autonomía médica y del valor del profesional de la salud frente a la sociedad ${ }^{14}$.

4. Las estructuras organizacionales rígidas y horas de trabajo inflexibles ${ }^{15}$.

5. La gran burocracia en los sistemas regulatorios médicos, que, en el caso colombiano, se traduce en acreditaciones, habilitaciones, certificaciones, convalidaciones e inspecciones de calidad, entre otros ${ }^{16}$.

6. El conocimiento médico, que hace que los doctores tengan un riesgo mayor que la población general de adicción o mal uso de medicamentos al automedicarse.

7. La tendencia médica de no buscar ayuda y soporte cuando no se sienten bien o están bajo presión ${ }^{17}$.

8. El estigma que tienen los doctores frente a la enfermedad mental ${ }^{18}$.

9. Los hábitos poco saludables de los cardiólogos: tabaquismo activo, sedentarismo... ${ }^{19}$.

10. El frecuente uso y abuso de sustancias entre los médicos ${ }^{20}$.

\section{La cardiología desde la perspectiva de la práctica clínica}

La cardiología es un área de la medicina que se destaca por los frecuentes avances tecnológicos y la investigación científica ${ }^{21}$. El ingreso a este privilegiado mundo de la medicina se vincula con mayores ingresos económicos y mayor prestigio derivado del hecho de pertenecer a una especialidad con alta valoración en el mundo médico y en la sociedad en general. Y si bien la cardiología puede ser una especialidad con grandes recompensas personales, también puede ser extenuante y demandante, lo cual puede conducir a un sindrome de "burnout",22.

El cardiólogo, además de la carga asistencial y la fatiga, enfrenta situaciones emocionales asociadas con sufrimiento, miedo, falla de los tratamientos, errores, muerte, interacción con familias y con otro personal médico, demandas y jueces. Toda esta demanda cognitiva generada en la necesidad de procesar rápidamente cantidades desbordantes de información por períodos largos puede afectar negativamente la salud y la calidad del trabajo del cardiólogo ${ }^{23}$.

\section{"Burnout" o síndrome de agotamiento profesional}

Este es un síndrome que resulta de la prolongada exposición a estrés ocupacional, y comprende tres componentes: 1) cansancio emocional como resultado de la carga excesiva, 2) despersonalización, la cual se refiere a un sentido de cinismo y carencia de compasión por los pacientes y pares; y 3) sentimiento de disminución del logro personal que captura un sentido de competencia y eficiencia profesional ${ }^{24}$.

Es común en los médicos de todas las especialidades y todos los escenarios de atención. En el primer gran estudio de agotamiento profesional en médicos realizado en el 2012, el $45,8 \%$ de 7.288 médicos reportó por lo menos un síntoma ${ }^{25}$. Sin embargo, el incremento en la prevalencia de este síndrome en los últimos años ha sido llamativo ${ }^{26}$.
En un estudio realizado por Peckham en el $2017^{27}$, los cardiólogos entre diversas especialidades médicas presentaban una prevalencia de "burnout" del 52\%; la más alta fue para los médicos de urgencias con $59 \%$ y la más baja para los infectólogos con 3,9\%. Además, se pudo observar que en el grupo de los cardiólogos fue mayor en mujeres que en hombres ( 55 vs. $51 \%$ ) y que las principales causas fueron las tareas burocráticas, demasiadas horas de trabajo, la computarización de la práctica, mantener la certificación, los ingresos, la mala práctica, la insatisfacción con el cuidado de los pacientes, el excesivo número de pacientes en un día, las dificultades con el empleador, los colegas y el staff; los pacientes difíciles, la sobreexposición a la muerte de pacientes, la incapacidad para mantenerse actualizado $y$, en el último lugar, el estrés familiar.

\section{Factores que contribuyen al agotamiento profesional}

Numerosos factores contribuyen a la aparición de burnout en cardiólogos: carga laboral excesiva y desbalance entre las demandas del trabajo, las habilidades y la pérdida de control $^{28}$. Las cinco causas más reportadas por los cardiólogos en el Medscape Cardiologist Lifestyle Report 2017 fueron la gran carga de tareas burocráticas, la cantidad de tiempo gastado en el trabajo, el incremento de prácticas computarizadas, la pérdida de autonomía y las exigencias para alcanzar certificaciones.

\section{Consecuencias del agotamiento profesional}

Esta condición afecta adversamente a los médicos, los pacientes y el sistema de salud, y es por esto que se propone como un indicador de la calidad de la asistencia en salud.

Así mismo, se ha encontrado una fuerte asociación entre burnout y enfermedad mental, abuso de sustancias y suicidio en médicos. De acuerdo con la Fundación Americana para la Prevención del Suicidio, la tasa de suicidio de los médicos con burnout es mayor, particularmente en las mujeres ${ }^{29}$. De igual forma, el alcohol y el abuso de sustancias es más común en médicos que en la población general (10-15 vs. 8,0\%) y se asocia con burnout ${ }^{30}$.

Por otra parte, se ha observado que los médicos con burnout ofrecen menor cuidado a sus pacientes. Una revisión sistemática y un metaanálisis recientes apoyan esta asociación, además de una menor satisfacción en el paciente atendido por médicos con burnout ${ }^{31}$.

\section{Depresión y ansiedad en médicos}

Se han descrito altas prevalencias de trastorno depresivo en médicos ${ }^{32}, y$, adicionalmente, se ha encontrado asociación entre depresión y estrés laboral ${ }^{33}$.

La prevalencia de depresión en hombres médicos, reportada en una cohorte de médicos de sexo masculino graduados entre 1948 y 1964 , fue del $12,8 \%{ }^{34}$, mientras que en un gran estudio que incluyó 4.501 médicas fue del $19,5 \%{ }^{35}$; sin embargo, estas prevalencias no difieren sustancialmente de las encontradas en población general, tanto 
TRASTORNOS NEURODEGENERATIVOS

Menor neurogénesis, disfunción cerebrovascular, APOPTOSIS

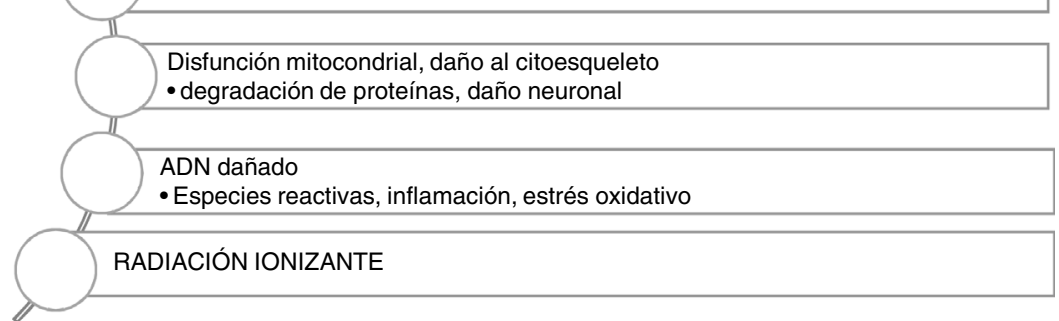

Figura 1 Radiación ionizante, daño neuronal y enfermedades neurodegenerativas (Elaboración propia).

para hombres como para mujeres ${ }^{36}$, sin que deje de ser importante que una de cada cinco médicas y uno de cada diez médicos pueden sufrir de depresión en algún momento de sus vidas.

\section{Resumen de la relación entre la profesión médica, la cardiología y el riesgo para la salud mental}

La cultura médica ha otorgado una baja prioridad a la salud mental a pesar de la evidencia contundente que demuestra que los trastornos mentales, el burnout y los problemas de alcohol y de sustancias no tratados, incrementan el riesgo suicida y los errores médicos, y disminuyen sustancialmente la calidad de vida y la productividad laboral.

Las barreras que impiden a los médicos buscar ayuda necesitan ser superadas, al igual que las actitudes profesionales y las políticas institucionales requieren ser cambiadas. Los estudiantes de medicina y los médicos precisan aprender a buscar ayuda para problemas de salud mental.

\section{Exposición ocupacional a bajas dosis de radiación y enfermedad mental}

El sistema nervioso central es sensible a una variedad de noxas, entre las cuales se encuentran el trauma, la isquemia, la depresión, la quimioterapia y la radiación ionizante. La exposición a radiación ionizante permite un amplio espectro de lesiones en diferentes estructuras celulares a partir del estrés celular que compromete el metabolismo celular, la reparación del ADN, la progresión del ciclo celular y la supervivencia celular; además, genera inflamación y estrés oxidativo $^{37,38}$.

En la actualidad es claro que la exposición a altas dosis de radiación ( $\geq 20 \mathrm{~Gy}$ ) inhibe los procesos de neurogénesis ${ }^{39}$. Adicionalmente, esa exposición a dosis mayores ( $\geq 45 \mathrm{~Gy}$ ) se asocia con inicio y progresión de déficit cognitivo ${ }^{40,41}$. No obstante, menos estudiados han sido los efectos de la exposición a bajas dosis de radiación ( $\leq 2 \mathrm{~Gy}$ ), lo cual se encuentra típicamente en exposiciones ambientales, ocupacionales o diagnósticas ${ }^{42,43}$.

Recientemente se ha demostrado que el sistema nervioso central, que había sido considerado por largo tiempo un sistema radiorresistente, es susceptible incluso a bajas dosis de radiación. Esta susceptibilidad parece depender de la baja capacidad antioxidante, la alta tasa metabólica y la presencia de células que se dividen activamente en dos regiones del cerebro (el giro dentado del hipocampo y la zona subventricular) ${ }^{44}$.

En un experimento realizado con monos, en el cual se compararon grupos con radiación y otros sin ella, se observó hiperactividad motora dependiente de la dosis de radiación, así como cambios en las tareas afectivas en términos de comportamientos ansiosos ${ }^{46}$.

Además de la inhibición de la neurogénesis por la radiación (dosis-dependiente) y de la neuroinflamación que esto implica ${ }^{45,46}$, la exposición a bajas dosis de radiación incrementa las especies reactivas de oxígeno y nitrógeno, las cuales tienen el potencial de alterar el balance de reducción del SNC, aumentar el estrés oxidativo, generar disfunción mitocondrial y permitir la degradación de proteínas, todo lo cual llevaría a envejecimiento celular y muerte por apoptosis ${ }^{47,48}$. Todos estos procesos están implicados en enfermedades neurodegenerativas y psiquiátricas (fig. 1).

La radiación daña el $A D N$, al generarle rupturas que impiden la replicación de este en células en crecimiento, y causa la detención en la fase $\mathrm{S}$ del ciclo de desarrollo. Además, conduce a la formación de especies reactivas de oxígeno (ROS) que causan estrés oxidativo en las células y estas además dañan indirectamente el ADN, al desregular el ciclo celular, dañar las proteínas y los lípidos al oxidarlos y eventualmente producir la muerte celular. Ya que el cerebro tiene baja replicación celular, las lesiones repetidas y no reparadas en el ADN se acumulan, especialmente cuando la exposición es a bajas dosis de radiación $1<50$ cGy) ${ }^{49}$. Por tanto, los efectos de la exposición a bajas dosis de radiación en el cerebro son múltiples, y entre ellos se encuentran:

1. Cambios morfológicos y funcionales en el tejido cerebral $^{50}$.

2. Cambios en la vascularización ${ }^{51}$.

3. Disminución de la proliferación y neurogénesis en el hipocampo ${ }^{52}$.

4. Decline cognitivo con déficit de aprendizaje y memoria ${ }^{53}$.

5. Alteración de la señalización en el hipocampo y el lóbulo frontal ${ }^{54}$. 


\section{Desregulación de micro ARN-134 en cardiólogos intervencionistas expuestos a bajas dosis de radiación}

Los microARN (miARN) son una clase de moléculas de ARN cortas, no codificantes, de aproximadamente 22 nucleótidos, las cuales han sido encontradas desreguladas en numerosas enfermedades humanas ${ }^{55}$. Por su estabilidad y especificidad tisular los miARN son biomarcadores ideales para explorar los mecanismos moleculares que subyacen y están asociados con exposición a bajas dosis de radiación. En un estudio reciente, de tres etapas (descubrimiento, selección y validación), se quiso estudiar los miARN de cardiólogos intervencionistas. En la primera etapa participaron 10 cardiólogos intervencionistas, 6 de ellos hombres con una edad promedio de 54 años (DE 5,4) y 10 controles no expuestos, pareados por sexo y edad. En la etapa de selección todos los miARN desregulados fueron evaluados en 80 muestras de plasma (40 de cardiólogos intervencionistas y 40 sujetos no expuestos) y en la tercera etapa los miARN identificados fueron investigados en una cohorte de 132 cardiólogos intervencionistas, 102 hombres con una edad promedio de 46,3 años (DE 9,0) y 83 sujetos no expuestos (51 hombres con una edad de 47,9 años (DE 8,0 años). El análisis finalmente identificó dos diferentes miARN (miR-134 y miR-2392), expresados en los cardiólogos intervencionistas, regulados a la baja y con diferencias significativas al compararlos con los sujetos no expuestos ${ }^{56}$. El miR-134 ha sido asociado con desregulación en varios cánceres, actuando como supresor y promotor de tumores ${ }^{57}$, en tanto que el miR2392 ha sido asociado con progresión de cáncer gástrico ${ }^{58}$.

\section{Propuestas para mitigar el agotamiento profesional y promover el bienestar de los cardiólogos}

Cuando los médicos no están bien, no se sienten a gusto, están agotados física y mentalmente, los desempeños de los sistemas de salud pueden ser subóptimos. El bienestar de los médicos no solo beneficia al médico de forma individual, sino que puede ser un factor esencial para brindar cuidados de salud de alta calidad.

El significado de la palabra bienestar puede que no abarque apropiadamente todo lo que se pretende nombrar con ella: salud física, mental, emocional y bienestar. El bienestar puede trascender el hecho de no tener distrés para incluir aspectos como alcanzar metas personales y profesionales. Sin duda la profesión médica es estresante y pesada. Prueba de esto es que el $64 \%$ de los médicos canadienses reportaron que sentían que sus cargas laborales eran demasiado pesadas y $48 \%$ afirmaban que estas habían incrementado en el año pasado. Además, los estudios muestran de manera consistente, que los médicos trabajan entre 50 y 60 horas por semana cuando no están de llamada ${ }^{59}$, lo cual, sin duda, supera la jornada laboral de la mayoría de trabajadores de otras áreas.

Parece clara la necesidad de abordar este importante tema con una mirada desde la promoción de estilos de vida más saludables y desde la prevención, que permita que los síntomas se detecten temprano y el curso y pronóstico de los trastornos mentales sea mejor. A continuación se presentan algunas de las estrategias que se han propuesto al respecto.

- Bienestar médico como una responsabilidad de los sistemas de salud y de los individuos: el sistema de salud requiere reformas profundas tales como la reducción de las tareas administrativas de los médicos, modelos de compensación, nuevos modelos de práctica, equipos de trabajo, soporte financiero y tiempo protegido para que los médicos se vinculen con los programas de bienestar. Se espera que los cardiólogos practiquen un mayor autocuidado (ejercicio, descanso, sueño) ${ }^{60}$.

- Crear una nueva cultura: las escuelas de medicina y las asociaciones profesionales necesitan enseñar nuevos valores a los cardiólogos tales como la importancia del autoconocimiento, el balance entre la vida y el trabajo, la aceptación clínica de la incertidumbre, buscar ayuda para problemas mentales. Esto se podría lograr implementando módulos de balance entre vida y trabajo, entrenamiento en mindfulness para el manejo del estrés y como actitud positiva frente a la vida, y mentorías para los médicos más jóvenes $^{61}$.

Valorar y fortalecer la resiliencia individual y organizacional: es necesario reconocer, construir y aumentar las características positivas de las instituciones de salud y los cardiólogos $^{62}$.

Vincular líderes institucionales e individuales que ayuden a vincular precisamente a aquellos que más lo necesitan puesto que existe evidencia que indica que quienes tienen mayor burnout son aquellos que más se resisten a este tipo de iniciativas ${ }^{63}$.

- Desarrollar nuevas herramientas para capturar la naturaleza multidimensional del bienestar médico: se necesitan instrumentos psicométricos que se validen y se usen masivamente, para conocer mejor el comportamiento de los cardiólogos frente al constructo llamado bienestar ${ }^{64}$.

\section{Trastornos mentales y exposición ocupacional a bajas dosis de radiación}

Los estudios de exposición ocupacional o accidental a radiación y enfermedad mental, son de gran interés, pero la evidencia es limitada, fragmentaria, en algunos casos conflictiva y con fallas metodológicas, por tanto, la evidencia disponible es insuficiente para arrojar conclusiones. A continuación, se presenta la información disponible en la actualidad.

Existe acuerdo en que los efectos de la radiación son más pronunciados en niños. Estos pueden perder parte de sus habilidades cognitivas y tener un grave decline del coeficiente intelectual, el cual parece estar relacionado con el nivel cognitivo previo y una menor edad al momento de la exposición ${ }^{65}$. En los adultos se ha propuesto que los efectos agudos de la radiación son problemas en la memoria semántica, por daño del hipocampo ${ }^{66}$.

En cuanto a la asociación entre radiación y demencia, los tiempos de seguimiento en los diferentes estudios han sido menores de diez años ${ }^{67}$. Algunos estudios como el realizado 
con población de Rochester, no encontró asociación entre radiación y demencia de tipo Alzheimer ${ }^{68}$.

\section{Depresión y ansiedad}

En los pocos estudios que han explorado la posible incidencia de depresión luego de radioterapia, no se ha encontrado relación ${ }^{62}$, sin embargo, con los conocimientos actuales de la neurobiología de la depresión se sabe que el compromiso del hipocampo interfiere con procesos de memoria, aprendizaje, regulación emocional y respuesta al estrés, que se sabe también se comprometen en la depresión ${ }^{69}$.

En la última década se ha propuesto que diferentes trastornos psiquiátricos, incluidos la depresión, el trastorno bipolar y la esquizofrenia, podrían estar relacionados con una desregulación en algunas vías de la neurogénesis, particularmente a nivel del hipocampo. Incluso algunos psicofármacos, como los antidepresivos y los estabilizadores del afecto, ejercen parte de su efecto al mejorar la supervivencia y la plasticidad neuronal en el hipocampo ${ }^{70}$. Por lo tanto, es plausible que la reducción de la neurogénesis después de la radiación pueda predisponer a personas vulnerables al comienzo de un trastorno mental y afectar negativamente el curso de los síntomas y el pronóstico del tratamiento ${ }^{58}$.

En un estudio transversal, el cual incluyó 746 cuestionarios que fueron diligenciados por 466 personas expuestas a radiación ionizante, 218 eran cardiólogos intervencionistas o electrofisiólogos con una edad de 45 años \pm 9 años, con una mediana de tiempo de trabajo de 10 años (cuartiles 524). La prevalencia de problemas ansiosos y depresivos fue de $12,4 \%$ en el grupo expuesto y de $2,1 \%$ en el no expuesto, con una diferencia significativa $(p<0,001)^{71}$.

En un estudio que incluyó 1.621 taiwaneses expuestos a radiación en sus hogares por construcciones reforzadas con barras contaminadas, se aplicó el inventario de Beck (BDI) que tamiza riesgo de depresión y se encontró que la prevalencia de depresión en las personas expuestas fue de $18,7 \%$, con mayor riesgo para aquellos con mayor exposición [OR 1,46 (IC 95\% 1,02-2,07)] y que tenían historia previa de depresión [OR 2,49, (IC 95 1,36-4,58)] $]^{72}$.

\section{Exposición a radiación y trastornos del espectro de la esquizofrenia}

También se dispone de alguna evidencia en cuanto a un incremento en la incidencia del espectro de los trastornos relacionados con la esquizofrenia, luego de la exposición a la radiación de bombas atómicas o radioterapia ${ }^{73}$. De hecho, se ha reportado un aumento en la incidencia de los trastornos relacionados con la esquizofrenia en los sobrevivientes de Nagasaki. La prevalencia fue de $6 \%$, significativamente más alta que el $1 \%$ encontrado en la población general ${ }^{74}$, sin embargo, este estudio tuvo importantes limitaciones metodológicas que afectan la veracidad de estos hallazgos. Luego del desastre de Chernobyl se reportó un aumento en la incidencia de esquizofrenia en los sobrevivientes, lo cual se explicó como una consecuencia de la disfunción frontotemporo-límbica izquierda ${ }^{75,76}$.

En un estudio reciente que evaluó la posible asociación entre exposición a radiación y esquizofrenia, un grupo de más de 10.000 sujetos expuestos a radioterapia en la cabeza durante la niñez para tratamiento de tiña capitis, no se encontró mayor incidencia de esquizofrenia que en el grupo control $^{77}$.

Yamada et al. ${ }^{78}$ no encontraron relación entre la exposición a altas y bajas dosis de radiación en 2.286 sobrevivientes de la bomba atómica y enfermedad mental. En otro estudio se halló un incremento en la proporción de mujeres blancas y técnicas de rayos $X$ con muerte por demencia tipo Alzheimer, comparado con trabajadoras de otras ocupaciones ${ }^{79}$.

\section{Compromisos neuropsicológicos en cardiólogos intervencionistas}

En las últimas dos décadas se ha presentado un interés especial por determinar los compromisos neuropsicológicos asociados a exposición por largos períodos de tiempo a bajas dosis de radiación, como ocurre en cardiólogos intervencionistas. Los hallazgos más consecuentes muestran una reducción significativa en la memoria, principalmente en la memoria verbal de largo plazo y la fluencia verbal, ambos desempeños atribuidos a habilidades del hemisferio izquierdo, que se verían comprometidos con la exposición a bajas dosis de radiación ${ }^{80}$.

Finalmente, son pocos los datos al respecto y se necesitan nuevos y mejores estudios que permitan determinar si existe un vínculo entre exposición a bajas dosis de radiación y mayor riesgo de sufrir trastornos mentales.

\section{Creative commons}

Creative Commons Reconocimiento-No Comercial-Sin Obra Derivada (CC BY-NC-ND).

\section{Conflicto de intereses}

Ninguno.

\section{Bibliografía}

1. World Health Organization WHO. National health workforce accounts: a handbook. Geneva: WHO; 2016.

2. Carrieri D, Briscoe S, Jackson M, Mattick K, Papoutsi C, et al. Care Under Pressure: a realist review of interventions to tackle doctor's mental ill-health and its impacts on the clinical workforce and patient care. BMJ Open. 2018;8:e021273.

3. World Health Organization (WHO). Health workforce-data and statistics. Geneva: WHO; 2016 2016. [Acceso 12 Dic 2018]. Disponible en: http://www.who.int/hrh/statistics/en/.

4. Kreitzer MJ, Klatt M. Educational innovations to Foster resilience in the heath profession's. Med Teach. 2017;39:153-9.

5. Wallace JE, Lemaire JB, Ghali WA. Physician wellness: a missing quality indicator. Lancet. 2009;374:1714-21.

6. Shanafelt TD, Hasan O, Dyrbye LN, Sinsky C, Satele D, et al. Changes in burnout and satisfaction with work-life balance in physicians and the General US Working Population Between 2011 and 2014. Mayo Clin Proc. 2015;90:1600-13.

7. Hayes B, Prihodova L, Walsh G, Doyle F, Doherty S. What's up doc? A national cross-sectional study of psychological wellbeing of hospital doctors in Ireland. BMJ Open. 2017;7:e018023. 
8. Hampton T. Experts address risk of physician suicide. JAMA. 2005;294:1189-91.

9. Bodeneimer T, Sinsky C. From triple to quadruple aim: care of the patient requires care of the provider. Ann Fam Med. 2014;12:573-6.

10. Limb M. Stress levels of NHS staff are "astronishingly high" and need treating as a public health problem, sys King's Fund. BMJ. 2015;351:h6003.

11. House of Lords. The long-term Sustainability of the NHS and adult social care. [Internet]. HL paper 151. London: The House, 2017. p 35. [Acceso 12 Dic 2018]. Disponible en: https://publications.parliament.uk/pa/ld201617/ldselect/ Idnhssus/151/151.pdf.

12. Weinberg A, Creed F. Stress and psychiatric disorders in health care professional and hospital staff. Lancet. 2000;355:533-7.

13. Jones MC, Wells M, Gao C, Cassidy B, Davie J. Work stress and well-being in.

14. Freidson E. Doctoring together: A Study of Professional Social Control. New York: Elsevier; 1975.

15. Leape L, Berwick D, Clancy C, Conway J, Gluck P, et al. Lucian Leape Institute at the.

16. Department of Health. Mental health and ill health in doctors. [Internet]. London: DH; 2008. [Acceso 12 Dic 2018]. Disponible en: http://www.em-online.com/download/ medical_article/36516_DH_083090\%5B1\%5D.pdf.

17. Levine RE, Bryant SG. The depressed physician: a different kind of impairment. Hosp Phys. 2000;36:67-71.

18. Haque S. Stigma of mental health amongst physicians: one resident's experience about stigma in psychiatry among physicians, possible causes and a possible solution. Asian J Psychiatr. 2018;36:128-9.

19. Faggianoi P, Temporelli PL, Zito G, Bovenzi F, Colivicchi F, et al. Cardiovascular risk profile and lifestyle habits in a cohort of italian cardiologist Results of the SOCRATES survey. Monaldi Arch Chest Dis. 2013;80:118-25.

20. Srensen JK, Pedersen AF, Vedsted P, Bruun NH, Christensen B. Substance use disorders among Danish physicians: an explorative study of the professional socialization and management of colleagues with substance use disorders. J Addict Md. 2016;10:248-54.

21. Rodgers GP, Conti JB, Feinstein JA, Griffin BP, Kennett JD, et al. ACC 2009 survey results and recommendations: addressing the cardiology workforce crisis. A report of the ACC board of trustee's workforce task force. J Am Coll Cardiol. 2009;54:1195-208.

22. Michel JB, Sangha DM, Erwin JP. Burnout among cardiologist. Am J Cardiol. 2017;119:939-40.

23. Levin S, Aronsky D, Hemphill R, Han J, Slagle J, et al. Shifting toward balance: measuring the distribution of workload among emergency physician teams. Ann Emerg Med. 2007;50:419-23.

24. Maslach C, Schaufeli WB, Leiter MP. Job burnout. Annu Rev Psychol. 2001;52:397-422.

25. Shanafelt TD, Hasan O, Byrbye LN, Sinsky C, Satele D, et al. Changes in burnout and satisfaction with work-life balance in physicians and the general US working population between 2011 and 2014. Mayo Clin Proc. 2015;90:1600-13.

26. Panagioti M, Geraghty K, Johnson J. How to prevent burnout in cardiologist? Of the Current evidence, gaps, and future. Trends in Cardiovascular Medicine. 2018;28:1-7.

27. Medscape Cardiologist Lifestyle Report. 2017: race and ethnicity, bias and burnout. [Internet]. [Acceso 12 Dic 2018]. Disponible en: https://www.medscape.com/features/ slideshow/lifestyle/2017/cardiology\#page $=2$.

28. Shanaflt TD, Dyrbye LN, West CP. Addressing physician burnout the way forward. J Am Med Assoc. 2017;317:901-2.

29. Fridner A, Belkic K, Minucci D, Pavan L, Marini M, et al. Work environment and recent suicidal thoughts among male university hospital physicians in Sweden and Italy The health and organization among university hospital physicians in Europe (HOUPE) study. Gend Med. 2011;8:269-79.

30. Oreskovich MR, Shanafelt T, Dyrbye LN, Tan L, Sotile W, et al. the prevalence of substance use disorders in American Physicians. Am J Addiction. 2015;24:30-8.

31. Hall LH, Johnson J, Watt I, Tsipa A, OĆonor DB. Healthcare staff wellbeing, burnout, and patient safety: a systematic review. Plos One. 2016;11:e0159015.

32. Center C, Davis M, Detre T, Ford DE, Hansbrough W, et al. Confronting depression and suicide in physicians: a consensus statement. JAMA. 2003;289:3161-6.

33. Wada K, Yoshikawa T, Goto T, Hirai A, Matsushima E, Kakashima $Y$, et al. National survey of the association of depressive symptoms with the number of off duty and on-call, and sleep hours among physicians working in Japanese hospitals: a cross sectional study. BMC Public Health. 2010;12:127.

34. Ford DE, mead LA, Chang PP, Cooper-Patrick L, Wang NY, Klag MJ. Depression is a risk factor for coronary artery disease in men: the precursors study. Arch Intern Med. 1998;158:1422-6.

35. Frank E, Dingle AD. Self-reported depression and suicide attempts among US women physicians. Am J Psychiatry. 1999;156:1887-94.

36. Blazer DG, Kessler RC, McGonagle KA, Seartz MS. The prevalence and distribution of major depression in a national community simple: The National Comorbidity Survey. Am J psychiatry. 1994;151:979-86.

37. Chiang CS, Hong JH, Stalder A, Sun Jr, Whithers HR, MCBride WH. Delayed molecular responses to brain irradiation. Int J Radiat Biol. 1997; 72:45-53.

38. Parihar VK, Acharya MM, Roa DE, Bosch O, Christie LA, Limoli CL. Defining functional changes in the brain caused by targeted stereotaxic radiosurgery. Translational Cancer Res. 2014;3:124-37.

39. Tseng BP, Giedzinski E, Izadi A, Suarez T, Lan ML, Tran KK, et al. Functional consequences of radiation-induced oxidative stress in cultured neural stem cells and the brain exposed to charged particle irradiation. Antioxid Redox Signan. 2013;20:1410-22.

40. Belarki K, Jopson T, Arellano C, Fike Jr, Rosi S. CCR2 deficiency prevents neuronal dysfunction and cognitive impairments induced by cranial irradiation. Cancer Res. 2013;73:1201-10.

41. Greene-Schloesser D, Moore E, Robbins ME. Molecular pathways: radiation-induced cognitive impairment. Clinical Cancer Res. 2013;19:2294-300.

42. Trivedi R, Khan AR, Rana P, Haridas S, Kumar H, Manda K, et al. Radiation-induced early changes in brain and behavior: serial diffusion tensor imaging and behavior evaluation after graded doses of radiation. Journal of Neuroscience Research. 2012;90:2009-19.

43. Mothersill C. Radiation-induced bystander effects: past history and future directions. Radiat Res. 2001;155:759-67.

44. Manda K, Reiter RJ. Melatonin maintains adult hippocampal neurogenesis and cognitive functions after irradiation. Prog Neurobiol. 2010;90:60-8.

45. Acharya MM, Patel NH, Craver BM, Tran KK, Giedzinski E, Tseng BP, et al. Consequences of low dose ionizing radiation exposure on the hippocampal microenvironment. PloS One. 2015;10:e0128316.

46. Belarbi K, Jopson T, Arellano C, Fike JR, Rosi S. CCR2 deficiency prevents neuronal dysfunction and cognitive impairments induced by cranial irradiation. Cancer Res. 2013;73:1201-10.

47. Limoli CL, Giedzinski E, Rola R, Otsuka S, Palmer TD, Fike JR. Radiation response of neural precursor cells linking cellular sensitivity to cell cycle checkpoints, apoptosis and oxidative stress. Radiat Res. 2004;161:17-27.

48. Loganovsky K. Do low doses of ionizing radiation affect the human brain? Data Sci J. 2009;8:BR13-35.

49. Koturbash I, Jadavji NM, Kutanzi K, Rodriguez-Juarez R, Kogosov D, Metz GAS, Kovalchuk O. Fractionated low-dose exposure to ionizing radiation leads to DNA damage, epigenetic 
dysregulation, and behavioral impairment. Environ Epigenet. 2017;2:dvw025, 10.1093/eep/dvw025.

50. Limoli CL, Giedzinski E, Rola R, Otsuka S, Palmer TD, Fike JR. Radiation response of neural precursor cells: linking cellular sensitivity to cell cycle checkpoints, apoptosis and oxidative stress. Radiat Res. 2004;161:17-27.

51. Brown WR, Thore CR, Moody DM, Robbins ME, Wheeler KT. Vascular damage after fractionated whole-brain irradiation in rats. Radiat Res. 2005;164:662-8.

52. Wojtowicz JM. Irradiation as an experimental tool in studies of adult neurogenesis. Hippocampus. 2006;16:261-6.

53. Monje ML, Mizumatsu S, Fike JR, Palmer TD. Irradiation induces neural precursor-cell dysfunction. Nat Med. 2002;8:955-62.

54. Silasi G, Diaz-Heijtz R, Besplug J, Rodriguez-Juárez R, Titov V, Kolb B, et al. Selective brain responses to acute and chronic lowdose X-ray irradiation in males and females. Biochem Biophys Res Commun. 2004;324:1223-35.

55. Esteller M. Non-coding RNAs in human disease. Nat Rev Genet. 2011;12:861-74.

56. Borghini A, Vecoli C, Mercuri A, Capeggiani C, Piccaluga E, Guagilumi G, et al. Low-dose exposure to ionizing radiation deregulates the brain-specific microRNA-134 in interventional cardiologists. Circulation. 2017;136:2516-8.

57. Pan JY, Zhang F, Sun CC, Li SJ, Li G, Gong FY, et al. miR134: A Human Cancer Suppressor? Mol Ther Nucleic Acids. 2017;6:140-9.

58. J, Li T, Lu Y, Shen G, Guo H, Wu J, et al. MiR-2392 suppresses metastasis and epithelial-mesenchymal transition by targeting MAML3 and WHSC1 in gastric cancer. FASEB J. 2017; 31(9):377486.

59. ssociation. CMA Guide to physician health and well-being: facts, advice and resources for Canadian doctors [Internet]. Ottawa: Canadian Medical Association; 2003. [Acceso 12 Dic 2018]. Disponible en: http://www.mtpinnacle.com/pdfs/guidePHWB.pdf.

60. Panagioti M, Panagopoulou E, Bower P, Lewith g, Kontopantelis E, Chew-Graham C, et al. Controlled interventions to reduce burnout in physicians: a systematic review and meta-analysis. JAMA Intern Med. 2017;177:195-205.

61. Cass I, Duska LR, Blank SV, Cheng G, duPont NC, Frederick PJ, et al. Stress and burnout among gynecologic oncologists: a society of gynecologic oncology evidence-based review and recommendations. Gynecol Oncol. 2016;143:421-7.

62. Johnson J, Wood AM, Gooding P, Taylor PUJ, Tarrier N. Resilience to suicidality: the buffering hypothesis. Clin Psychol Rev. 2011;31:563-91.

63. Cheshire A, Hughes J, Ridge D, Panagioti M, peters D, Simon $C$, Lewith G. One size does not fit all: exploring general practitioner's perceptions of resilience training. $\mathrm{Br} \mathrm{J}$ Gen Pract. 2017;74:398-403.

64. Dyrbye LN, Trockel M, Frank E, Olson K, Linzer M, Lemaire J, et al. Development of a research agenda to identify evidencebased strategies to improve physician wellness and reduce Burnout. Ann Intern Med. 2017;166:743-4.

65. Schatz J, Ramer JH, Ablin, Mathay KK. Processing speed, working memory and IQ: a developmental model of cognitive deficits following cranial radiation therapy. Neuropyshcology. 2000;14:65-86.
66. Armstrong CL, Corn BW, Ruffer JE, Pruit AA, Mollman JE, Phillips PC. Radiotherapeutic effects on brain function double dissociation of memory systems. Neuropsychiatry Neuropsychol Behav Neuro. 2000;13:101-11.

67. Marazziti D, Baroni S, Catena-Dellósso M, Schiavi E, Ceresoli D, Conversano C, Picano E. Cognitive, psychological and psychiatric effects of ionizing radiation exposure. Current Medicinal Chemistry. 2012;19:1864-9.

68. Peper M, Steinvorth S, Scharaube P, Fruehauf S, Haas R, Kimmig $\mathrm{BN}$, et al. Neurobhavioral toxicity of total bodily irradiation. A follow up in long term survivors. Int J Radiat Oncol Biol Phys. 2000;46:303-11.

69. Hartung H, Tan SK, Steinbusch HM, Temel Y, Sharp T. High frequency stimulation of the sub thalamic nucleus inhibits the firing of juxta cellular labelled 5-hT containing neurons. Neuroscience. 2011;186:135-45.

70. Schumacher J, Jamra RA, Becker T, Ohlraun S, Klopp N, Binder $\mathrm{EB}$, et al. Evidence for a relationship between genetic variants at the brain-derived neurotrophic factor (BDNF) locus and major depression. Biol. Psychiatry. 2005;58:307-14.

71. Andreassi MG, Piccaluga E, Guagliumi G, Del Greco M, Gaita F, Picano E. Occupational health risks in cardiac catheterization laboratory workers. Circ Cardiovasc Interv. 2016;9:e003273.

72. Yen PN, Lin IF, Chang WP, Wang JD, Chag TC, Juo KL, et al. Risk factors of depression after prolonged low dose rate environmental exposure. Int J Radiat Biol. 2014;10:859-66.

73. Loganovsky KN, Volovik SV, Manton KG, Bazyka DA, Flor-Henry P. Whither ionizing radiation is a risk factor for schizophrenia spectrum disorders? World J Biol Psychiatry. 2005;6: 212-30.

74. Nakane $Y$, Ohta Y. An example from the Japanese register: some long term consequences of the A-bomb for its survivors in Nagasaki. En: Horn G, Giel R, Gulbinat WH, Henderson JH, editores. Psychiatric Case Registers in Public Health. Amsterdan: Elsevier; 1986. p. 26-7.

75. Loganovsky KN, Loganovskaja TK. Schizophrenia spectrum disorders in persons exposed to ionizing radiation as a result of the Chernobyl accident. Schizophr Bull. 2000;26: 751-73.

76. Nyagu Ai, Loganovsky KN, Loganovskaja TK. Psychophysiological after effect of prenatal irradiation. Int J Psychophysiol. 1998;30:303-11.

77. Sadetzki S, Chetri A, Mandelzweig L, Nahon D, Freedman L, Susser $\mathrm{E}$, et al. Childhood exposure to ionizing radiation to the head and risk of schizophrenia. Radiat Res. 2011;176:670-7.

78. Amada M, Sasaki H, Mimori Y, Kasagi F, Sudoh S, Ikeda J, et al. Prevalence and risks of dementia in the Japanese population: RERF's adult health study Hiroshima subjects Radiation Effects Research Foundation. J Am Geriatr Soc. 1999;47: 189-95.

79. Schulte PA, Burnett CA, Boerniger MF, Johnson J. Neurodegenerative diseases: occupational occurrence and potential risk factors, 1982 through 1991. Am J Public Health. 1996;86:1281-8.

80. Marazziti D, Tomaiuolo F, Dell’Osso L, Demi V, Campana S, Piccaluga $E$, et al. Neuropsychological testing in interventional cardiology staff after long-term exposure to ionizing radiation. J Int Neuropsychol Soc. 2015;21:670-6. 